\title{
In vitro antifungal effect of eugenol in combination with fluconazole against Candida sp.
}

\author{
Anek Pootong ${ }^{1 *}$, Kanokwan Wongdech ${ }^{1}$, Thitaporn Changrua ${ }^{1}$ and Narissara Mungkornkeaw ${ }^{2}$ \\ ${ }^{1}$ Department of Medical Technology, Faculty of Allied Health Sciences, Thammasat University \\ ${ }^{2}$ Bacteriology Unit, Medical Technology Laboratory, Thammasat University Hospital, Thammasat University, Pathum \\ Thani, Thailand. \\ Email: anek.p@allied.tu.ac.th
}

Received 30 September 2018; Received in revised form 1 July 2019; Accepted 4 July 2019

\begin{abstract}
Aims: A combination of the antimicrobial drug with the herbal derived antifungal agent was exploited as alternative therapeutic approaches for infectious diseases caused by drug resistant strains. In this study, we determine the antifungal effects of eugenol alone and in combination with fluconazole against Candida sp.

Methodology and results: Candida strains including fluconazole resistant (C. parapsilosis ATCC 22019 and C. albicans U821/10) and susceptible strains (C. tropicalis U624/10 and C. glabrata U71/1) were used in this study. By broth microdilution technique, eugenol exhibited antifungal activity with MIC and MFC against Candida sp. tested ranging from $0.5-1 \mathrm{mg} / \mathrm{mL}$. The interaction between eugenol and fluconazole against Candida sp. was determined by chequerboard microtiter technic. Eugenol decreased the MIC of fluconazole against Candida sp. tested. No antagonism was observed in strains test.
\end{abstract}

Conclusion, significance and impact of study: From these results, eugenol displayed a promising antifungal effect alone as well as combination with fluconazole against Candida sp.

Keywords: Eugenol, fluconazole, Candida, drug combination

\section{INTRODUCTION}

Candida is a commensal yeast found in the human skin and mucosal membrane surfaces. However, it was found as common opportunistic fungi causing infection of oral, vaginal, and systemic candidiasis. C. albicans is the predominant causative organism of almost types of candidiasis, but other emerging Candida sp. including $C$. glabrata, C. krusei, C. tropicalis, and C. parapsilosis are now posing serious nosocomial threats to patient populations (Chakrabarti et al., 2009; Xiao et al., 2018). Amphotericin B and fluconazole have been the drug of choice in the treatment of these fungal infections. However, amphotericin B is high nephro- and hepatotoxicity (Groll and Kolve, 2004). Due to its high solubility, low toxicity, and wide tissue distribution, fluconazole is the widely used for systemic candidiasis (Brammer et al., 1990). But fluconazole failures in the treatment of candidiasis have been observed due to intrinsically resistant Candida sp. such as $C$. krusei and C. glabrata and acquired resistant strains of $C$. albicans (Sobel et al., 2003). New therapeutic strategies are required to overcome these problems. The efficacy of antifungal agents including fluconazole can be improved by using drug combination therapy (Mukherjee et al., 2005). Such therapy has potential advantages over monotherapy in terms of reducing dose-related toxicity and emergence of drug resistance. Eugenol, a major active ingredient of clove oil, have been found to have the antifungal activity against Candida sp. with low cytotoxicity (He et al., 2007). In this study, we evaluated the antifungal effect of eugenol in combination with fluconazole against Candida sp. in vitro.

\section{MATERIALS AND METHODS}

\section{Candida sp.}

Besides one standard laboratory strain $C$. parapsilosis ATCC 22019 used as a quality control, clinical strain $C$. tropicalis (U624/10), C. glabrata (U71/1) and C. albicans (U821/10) were used in this study. Clinical strains were obtained from a urine sample in pure culture at $>10^{4}$ $\mathrm{CFU} / \mathrm{mL}$ were collected at Microbiology Laboratory Unit, Thammasat University Hospital. The strains were identified by using Brilliance ${ }^{\mathrm{TM}}$ Candida Chromogenic agar (Oxoid, Hampshire, UK) and sequencing of the internal transcribed spacer (ITS) of ribosomal DNA. The 
strains were cultured on Sabouraud dextrose agar (SDA) (Oxoid, Hampshire, UK) and incubated at $37^{\circ} \mathrm{C}$ for $48 \mathrm{~h}$ before use.

\section{Determination of minimum inhibitory concentration (MIC) and minimum fungicidal concentration (MBC) of eugenol and fluconazole}

Eugenol (Fluka, Steinhein, Germany) and fluconazole (Sigma-Aldrich, Missouri, USA) were used in this study. Eugenol or fluconazole was diluted in 1\% DMSO and used in the assay. The minimum inhibitory concentration (MIC) and minimum fungicidal concentration (MFC) of eugenol and fluconazole against Candida sp were determined by broth microdilution technic according to EUCAST (EDef 7.1) (Rodriguez-Tudela et al., 2008). Briefly, yeast suspension was prepared in sterile $0.85 \%$ normal saline solution (NSS) and then the concentration was adjusted to $5 \times 10^{5} \mathrm{CFU} / \mathrm{mL}$ in sterile distilled water. The compound was diluted twofold in double-strength RPMI 1640 (Biochrom AG, Germany) with 4\% glucose and $2 \%$ DMSO as solvent to achieve a range of concentration from $0.25-8 \mathrm{mg} / \mathrm{mL}$ for eugenol and $16-512$ $\mu \mathrm{g} / \mathrm{mL}$ for fluconazole. An equal volume of yeast suspension $\left(5 \times 10^{5} \mathrm{CFU} / \mathrm{mL}\right)$ was added. Drug-free control and cell-free were included as growth and sterility control. The suspensions were incubated at $37^{\circ} \mathrm{C}$ for 24 h. Optical density at $630 \mathrm{~nm}$ of the suspension was determined using a microplate reader. For the fluconazole, the MIC was calculated based on the density of the growth control and defined as the lowest concentration that results in at least $50 \%$ reduction in growth compared with that of the drug-free growth control. MIC of eugenol was defined as the lowest concentration that inhibited visible growth. Sub-culturing the optically clear well was performed to determine the MFC. The experiments were performed in duplicate and repeated three times.

\section{Chequerboard microdilution assay}

The interaction of eugenol with fluconazole was evaluated using the chequerboard microdilution assay in 96 -well microtiter plates according to methods described elsewhere (Vitale et al., 2005). Briefly, eugenol or fluconazole were serially two-fold diluted in in doublestrength RPMI 1640 (Biochrom AG, Germany) with 4\% glucose and $2 \%$ DMSO to obtain four-times the final concentration to be achieved in the microtiter well. Furthermore, $50 \mu \mathrm{L}$ of each dilution of eugenol was added to the microtiter well plates in the vertical direction, while $50 \mu \mathrm{L}$ of each dilution of fluconazole was added in the horizontal direction. One hundred microliters of yeast suspension $\left(2.5 \times 10^{5} \mathrm{CFU} / \mathrm{mL}\right)$ was added to each well. Plates were incubated at $37^{\circ} \mathrm{C}$ for $24 \mathrm{~h}$. Optical density at $630 \mathrm{~nm}$ (OD630) of the suspension was determined using a microplate reader.

To assess the interaction of combinations of drugs the data obtained spectrophotometrically were further analyzed using the fractional inhibitory concentration index $(\mathrm{FICl})$. $\mathrm{FICl}$ was defined as the following equation:

$\mathrm{FICl}=\mathrm{FICE}+\mathrm{FIC}_{\mathrm{F}}$

Where $\mathrm{FIC}_{\mathrm{E}}=\mathrm{MIC}$ of eugenol in combination/MIC of eugenol alone

$\mathrm{FIC}_{\mathrm{F}}=\mathrm{MIC}$ of fluconazole in combination $/ \mathrm{MIC}$ of fluconazole alone

According to EUCAST (2000) a synergistic effect (SynE) is observed when $\mathrm{FICl}$ value $\leq 0.5$; an additive effect (AddE) when $0.5<\mathrm{FICl}$ value $\leq 1$; an indifferent effect (IndE) when $1<\mathrm{FICl}$ value $<2$ and an antagonistic effect (AntE) when $\mathrm{FICl}$ value $\geq 2$

\section{RESULTS}

Candida infection is becoming more difficult to treat with antibiotic monotherapy because of an emergence of drug resistant strain. Thus, an effective and safe antifungal agent is required. The present study was conducted to evaluate the efficacy of herbal derived eugenol against Candida sp. and analyzes their interaction with fluconazole.

\section{Minimum inhibition concentrations (MICs) and minimum fungicidal concentrations (MFCs) for eugenol and fluconazole against Candida sp.}

Different Candida sp. including C. tropicalis U624/10, C. glabrata U71/11, C. parapsilosis ATCC 22019 and C. albicans U821/10 were used in this study. The MIC for fluconazole against $C$. tropicalis U624/10, C. glabrata U71/11, C. parapsilosis ATCC 22019, C. albicans U821/10 were $128,128,1$ and $0.25 \mu \mathrm{g} / \mathrm{mL}$, respectively. This result is consistent to the previous study reported that $C$. tropicalis U624/10 and C. glabrata U71/11 were considered as resistance strains to fluconazole whereas C. parapsilosis ATCC 22019 and C. albicans U821/1 were susceptible strains (Wongdech et al., 2018). Azole binds to ferric ion moiety and inhibits the activity of $14 \alpha$ demethylase (Erg11), disrupting the ergosterol biosynthetic pathway. Accumulated toxic-intermediate $4 \alpha-$ methyl sterols alter membrane stability, permeability, and the action of membrane-bound enzymes. However, most of the azole drugs are fungistatic and fail to completely eliminate yeast, leading the acquired resistance of $C$. albicans to azoles.

In our study, MIC and MFC for eugenol against $C$. tropicalis U624/10, C. glabrata U71/11, and C. albicans U821/10 were $1 \mathrm{mg} / \mathrm{mL}$ and $0.5 \mathrm{mg} / \mathrm{mL}$ for $C$. parapsilosis ATCC 22019 (Table 1) indicating antifungal activity of eugenol against both fluconazole susceptible and resistant Candida sp. This result confirmed that eugenol is effective against resistance mechanisms exhibited by Candida sp. (Ahmad et al., 2010a; Khan et al., 2012). It has been proposed that eugenol, monohydric phenol with lipophilic nature, may enter between the fatty acyl chains of the membrane lipid bilayer, disturbing its fluidity and permeability (Latifah-Munirah et al., 2015) and also found 
to inhibit an ergosterol biosynthesis in Candida. (Ahmad et al., 2010b) Additional, eugenol also perturbed the activity of amino acid permeases in yeast especially Gap1p which plays a role in amino acid sensing in a protein kinase A (PKA)-mediated protein phosphorylation cascade, resulting in cell death and subsequent cytoplasmic leakage (Darvishi et al., 2013). Because of its different target from azole drug, eugenol is seemed to be useful against clinical resistant isolates of Candida sp.

Table 1: Minimum inhibition concentration (MIC) and minimum fungicidal concentration (MFC) for eugenol and fluconazole against Candida sp.

\begin{tabular}{|c|c|c|c|}
\hline \multirow[b]{2}{*}{ Microorganism } & \multicolumn{2}{|c|}{ MIC } & \multirow{2}{*}{$\begin{array}{c}\text { MFC } \\
\text { eugenol } \\
\text { (mg/mL) }\end{array}$} \\
\hline & $\begin{array}{c}\text { fluconazole } \\
(\mu \mathrm{g} / \mathrm{mL})\end{array}$ & $\begin{array}{l}\text { eugenol } \\
\text { ( } \mathrm{mg} / \mathrm{mL} \text { ) }\end{array}$ & \\
\hline $\begin{array}{l}\text { C. tropicalis } \\
\text { U624/10 }\end{array}$ & 128 & 1 & 1 \\
\hline $\begin{array}{l}\text { C. glabata } \\
\text { U71/11 }\end{array}$ & 128 & 1 & 1 \\
\hline $\begin{array}{l}\text { C. parapsilosis } \\
\text { ATCC } 22019\end{array}$ & 0.5 & 0.5 & 0.5 \\
\hline $\begin{array}{l}\text { C. albicans } \\
\text { U821/10 }\end{array}$ & 0.125 & 1 & 1 \\
\hline
\end{tabular}

Fluconazole susceptibility of Candida sp. in combination with eugenol

The fluconazole susceptibilities of Candida sp. in combination with eugenol were shown in Table 2 and
Figure 1. MIC of fluconazole in combination with eugenol was reduced, especially in resistant strains. $\mathrm{FICl}$ values for eugenol in combination with fluconazole against all Candida sp. tested ranged from 0.6 to 1 . It indicated the additive interaction between eugenol and fluconazole. Moreover, no antagonistic interaction was found. This result emphasized a previous study reported that the combination of eugenol with fluconazole reduced the MIC of fluconazole against Candida sp. (Ahmad et al., 2010a). Eugenol may increase the susceptibility of Candida sp. to the accumulated toxic-intermediate sterols, impair fluconazole export by drug efflux pump or increase the import of azoles through the perturbed membrane. However, our study did not demonstrate the synergistic interaction of eugenol with fluconazole against Candida isolates as reported in the previous study (Ahmad et al., 2010a; Khan et al., 2012). In addition to the differences in Candida isolates tested, we believed that the inconsistency may be due to the different procedure used in chequerboard microdilution assay. The previous study used the protocol according to CLSI in chequerboard microdilution assay, while we used the protocol suggested by EUCAST. Although, the EUCAST and CLSI protocol were found to produce comparable results for testing the active antifungal drug including fluconazole (Pfaller et al., 2014). The agreement between these methods used for drug interaction study was unknown. Thus, the comparison study of chequerboard microdilution assay using the method according to CLSI and EUCAST should be performed further.
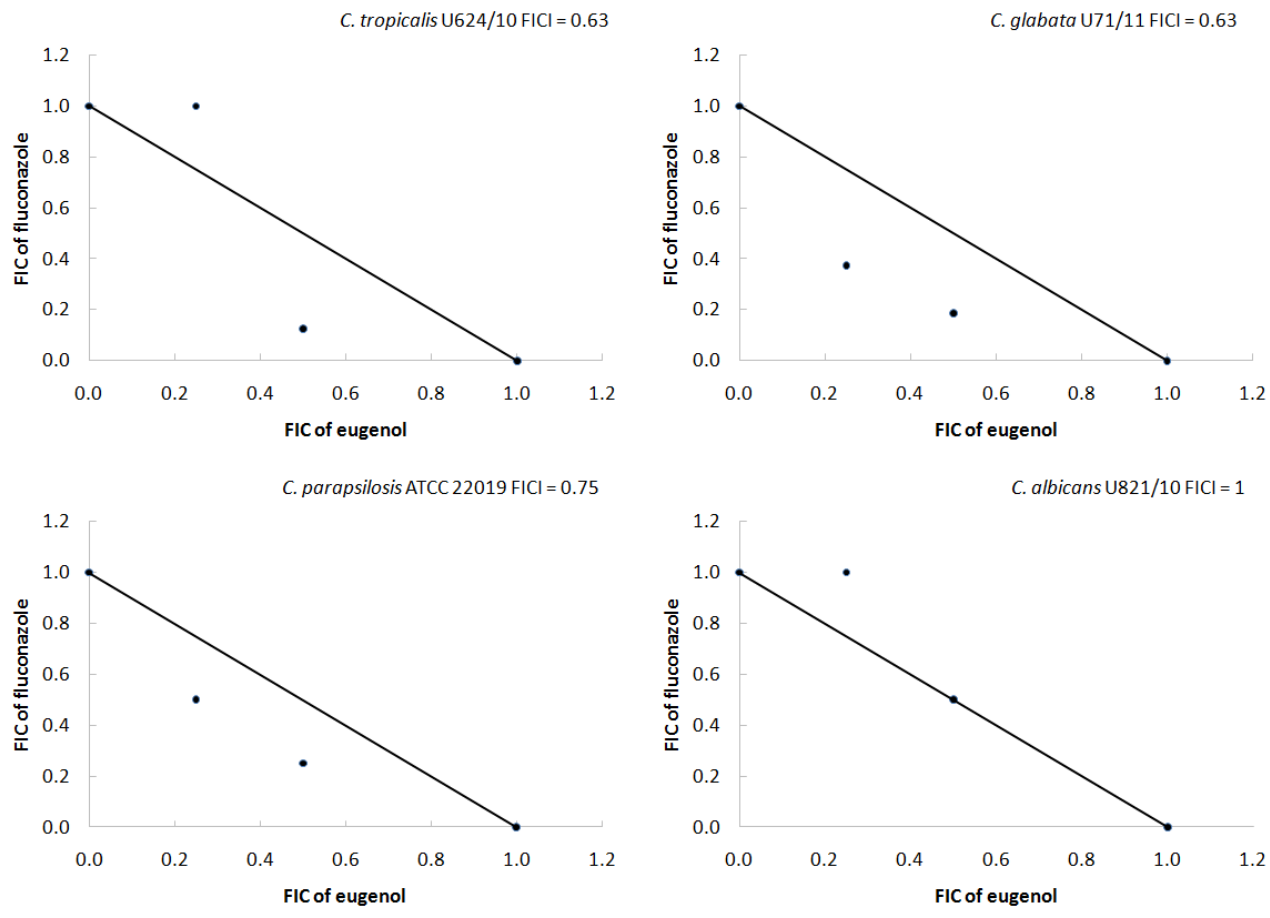

Figure 1: Isobologram of the FIC of eugenol and fluconazole against Candida sp. along with FICI values. 
Malays. J. Microbiol. Vol 15(7) 2019, pp. 518-522

DOI: http://dx.doi.org/10.21161/mjm.180256

Table 2: Susceptibilities of Candida strains used in this study to eugenol in combination with fluconazole.

\begin{tabular}{lllllll}
\hline Microorganism & Agent & $\mathrm{MIC}_{\mathrm{A}}$ & $\mathrm{MIC}$ & $\mathrm{FIC}$ & $\mathrm{FICl}$ & Interpretation \\
\hline C. tropicalis U624/10 & eugenol $(\mathrm{mg} / \mathrm{mL})$ & 1 & 0.5 & 0.5 & & \\
& fluconazole $(\mu \mathrm{g} / \mathrm{mL})$ & 128 & 16 & 0.13 & 0.63 & AddE \\
C. glabata U71/11 & eugenol $(\mathrm{mg} / \mathrm{mL})$ & 1 & 0.5 & 0.5 & & \\
C. parapsilosis & fluconazole $(\mu \mathrm{g} / \mathrm{mL})$ & 128 & 16 & 0.13 & 0.63 & AddE \\
ATCC 22019 & eugenol $(\mathrm{mg} / \mathrm{mL})$ & 0.5 & 0.25 & 0.5 & & AddE \\
C. albicans U821/10 & fluconazole $(\mu \mathrm{g} / \mathrm{mL})$ & 1 & 0.25 & 0.25 & 0.75 & \\
& eugenol $(\mathrm{mg} / \mathrm{mL})$ & 1 & 0.5 & 0.5 & & AddE
\end{tabular}

$\mathrm{MIC}_{\mathrm{A}}, \mathrm{MIC}$ of the agent alone; $\mathrm{MIC}_{\mathrm{C}}, \mathrm{MIC}$ of the agent in combination; AddE, Additive effect

\section{CONCLUSION}

In conclusion, these findings revealed that eugenol had the considerable antifungal activity against different species of Candida and also increased the susceptibility of Candida sp. to fluconazole. Antimicrobial activity against other common fungal pathogens must be studied in order to evaluate the potential of these compounds for therapeutic applications.

\section{ACKNOWLEDGEMENTS}

We would like to thank the Department of Medical Technology for technical support. We would also like to thank Associate Professor Dr. Worrada Samosornsuk for providing C. parapsilosis ATCC 22019 strain. Anek Pootong was financially supported by a research fund from the Faculty of Allied Health Sciences, Thammasat University in the Year 2018.

\section{REFERENCES}

Ahmad, A., Khan, A., Khan, L. A. and Manzoor, N. (2010a). In vitro synergy of eugenol and methyleugenol with fluconazole against clinical Candida isolates. Journal of Medical Microbiology 59, 1178-1184.

Ahmad, A., Khan, A., Manzoor, N. and Khan, L. A. (2010b). Evolution of ergosterol biosynthesis inhibitors as fungicidal against Candida. Microbial Pathogenesis 48, 35-41.

Brammer, K. W., Farrow, P. R., and Faulkner, J. K. (1990). Pharmacokinetics and tissue penetration of fluconazole in humans. Reviews of Infectious Diseases 12, S318-S326.

Chakrabarti, A., Chatterjee, S. S., Rao, K. L. N., Zameer, M. M., Shivaprakash, M. R., Singhi, S., Singh, R., and Varma, S. C. (2009). Recent experience with fungaemia: Change in species distribution and azole resistance. Scandinavian Journal of Infectious Diseases 41, 275-284.
Darvishi, E., Omidi, M., Bushehri, A. A., Golshani, A., and Smith, M. L. (2013). The antifungal eugenol perturbs dual aromatic and branched-chain amino acid permeases in the cytoplasmic membrane of yeast. PLOS ONE 8, e76028.

EUCAST. (2000). Terminology relating to methods for the determination of susceptibility of bacteria to antimicrobial agents. Clinical Microbiology and Infection 6, 503-508.

Groll, A. H., and Kolve, H. (2004). Antifungal agents: In vitro susceptibility testing, pharmacodynamics, and prospects for combination therapy. European Journal of Clinical Microbiology and Infectious Diseases 23, 256-270.

He, M., Du, M., Fan, M., and Bian, Z. (2007). In vitro activity of eugenol against Candida albicans biofilms. Mycopathologia 163, 137-143.

Khan, M. S. A., Malik, A., and Ahmad, I. (2012). Anticandidal activity of essential oils alone and in combination with amphotericin B or fluconazole against multi-drug resistant isolates of Candida albicans. Medical Mycology 50, 33-42.

Latifah-Munirah, B., Himratul-Aznita, W. H., and Mohd Zain, N. (2015). Eugenol, an essential oil of clove, causes disruption to the cell wall of Candida albicans (ATCC 14053). Frontiers in Life Science 8, 231-240.

Mukherjee, P. K., Sheehan, D. J., Hitchcock, C. A. and Ghannoum, M. A. (2005). Combination treatment of invasive fungal infections. Clinical Microbiology Reviews 18, 163-194.

Pfaller, M. A., Castanheira, M., Messer, S. A., Rhomberg, P. R., and Jones, R. N. (2014). Comparison of EUCAST and CLSI broth microdilution methods for the susceptibility testing of 10 Systemically active antifungal agents when tested against Candida spp. Diagnostic Microbiology and Infectious Disease 79, 198-204.

Rodriguez-Tudela, J. L., Arendrup, M. C., Barchiesi, F., Bille, J., Chryssanthou, E., Cuenca-Estrella, M., Dannaoui, E., Denning, D. W., Donnelly, J. P., Dromer, F., Fegeler, W., Lass-Flörl, C., Moore, C., 
Malays. J. Microbiol. Vol 15(7) 2019, pp. 518-522

DOI: http://dx.doi.org/10.21161/mjm.180256

Richardson, M., Sandven, P., Velegraki, A. and Verweij, P. (2008). EUCAST Definitive Document EDef 7.1: Method for the determination of broth dilution MICs of antifungal agents for fermentative yeasts. Clinical Microbiology and Infection 14, 398405.

Sobel, J. D., Zervos, M., Reed, B. D., Hooton, T., Soper, D., Nyirjesy, P., Heine, M. W., Willems, J. and Panzer, H. (2003). Fluconazole susceptibility of vaginal isolates obtained from women with complicated Candida vaginitis: Clinical implications. Antimicrobial Agents and Chemotherapy 47, 34-38.

Vitale, R. G., Afeltra, J. and Dannaoui, E. (2005). Antifungal combinations. Methods in Molecular Medicine 118, 143-152.

Wongdech, K., Changrua, T. and Pootong, A. (2018). In vitro antifungal effect of cinnamaldehyde in combination with fluconazole against Candida sp. Thai Science and Technology Journal 26, 1393-1400.

Xiao, M., Sun, Z. Y., Kang, M., Guo, D. W., Liao, K., Chen, S. C. A., Kong, F., Fan, X., Cheng, J.-W., Hou, X., Zhou, M. L., Li, Y., Yu, S.-Y., Huang, J. J., Wang, H. and $X u, Y$. C. (2018). Five-year national surveillance of invasive candidiasis: Species distribution and azole susceptibility from the China Hospital Invasive Fungal Surveillance Net (CHIF-NET) study. Journal of Clinical Microbiology 56, e0057700518. 